\title{
Laparoscopic Removal of Giant Gossypiboma
}

\author{
Federico Sista, MD, Malek Tabbara, MD, Christophe Barrat, MD, PhD, Sergio Carandina, MD \\ Service de Chirurgie Digestive et Métabolique-Hôpital Jean Verdier Bondy France, Université Paris XIII, Paris, France \\ (all authors). \\ Università degli Studi di L'Aquila, L'Aquila, Italy (Dr. Sista).
}

\begin{abstract}
Background: The term gossypiboma denotes a mass of cotton that is accidentally retained in the body postoperatively.

Case Report: Our case describes a 40-year-old female patient with a giant abdominal mass that occurred 9 years after a cesarean section delivery. Computed tomography scan and magnetic resonance imaging showed a 20-cm mass. A laparoscopic retrieval was performed with the placement of trocars on the previous Pfannenstiel incision scar. The patient was discharged 48 hours after surgery without complications.
\end{abstract}

Discussion: Gossypibomas are not rare, are often asymptomatic, and can be laparoscopically treated. To date, the literature has described different cases and stressed retention time and size as being limitations of laparoscopic retrieval.

Conclusion: To our knowledge, this is the first case of giant gossypiboma treated by laparoscopic suprapubic access. This approach permitted a reduction in complication rates, a shorter hospitalization time, and more satisfactory cosmetic results.

Key Words: Gossypiboma, Retained gauze, Surgical sponge, Foreign body.

Citation Sista F, Tabbara M, Barrat C, Carandina S. Laparoscopic removal of giant gossypiboma. CRSLS e2014.00032. DOI: 10.4293/CRSLS.2014.00032.

Copyright (C) 2014 SLS This is an open-access article distributed under the terms of the Creative Commons Attribution-Noncommercial-ShareAlike 3.0 Unported license, which permits unrestricted noncommercial use, distribution, and reproduction in any medium, provided the original author and source are credited.

Address correspondence to: Federico Sista, MD., Department of Metabolic and Digestive Surgery, Jean Verdier Hospital, 14 Juillet Blvd., 93140 Bondy, France

Telephone: (+33) 07-77-93-40-90, E-mail address: silversista@gmail.com.

\section{INTRODUCTION}

The term gossypiboma is used to describe surgical sponges or gauze retained inside a patient's abdomen after a surgical procedure. ${ }^{1-5}$ Gossypiboma is an infrequent but serious preventable condition that may lead to medico-legal problems. This clinical condition is often asymptomatic in the beginning and signs emerge latently, by the presence of an abdominal mass. ${ }^{6-8}$ Computed tomography scan and magnetic resonance imaging can identify this problem and aid in planning for surgical management. $3,5,9,10$ Surgical management of gossypiboma is a debated issue in the literature. $.^{2,7}, 10-13$ The laparoscopic approach has been described but could be associated with some limitations depending on the retention time and size of the sponges used., ${ }^{2,11,14}$ To our knowledge, this is the first case report of a $20 \mathrm{~cm}$-diameter gossypiboma that presented 9 years after a cesarean section delivery, which was found to have been caused by a laparoscopic sponge. Despite the size of the gossypiboma, we opted for laparoscopic removal through suprapubic access. This permitted us a safe and effective management of the clinical case, without further additional scars.

\section{CASE REPORT}

A 40-year-old woman was admitted to our surgical unit complaining of colicky abdominal pain and constipation that had been present for the previous 3 months and were increasing in intensity, without nausea or vomiting. Physical examination revealed the presence of a giant abdominal mass in the epimesogastric region, which was floating and painful on palpation, but neither was associated with weight loss or asthenia. Blood test results were unremarkable and tumor markers (CEA, CA19-9, CA125, CA15-3, aFP) were negative. She had no prominent past medical history, except for a tuberculous cervical lymphadenitis under a 4-month antibiotic treatment and 3 cesarean section (C-section) deliveries, the last one performed 9 years prior. The patient reported the appearance of the mass 
several months after the last C-section and noticed a slow increase in its size during the following years.

A contrast-enhanced abdominal computed tomography scan showed the presence of a voluminous cystic formation $(11 \times 12 \times 20 \mathrm{~cm})$ of a nonhomogeneous content with an estimated $30 \%$ vascular and calcific component with an external high-density wall. There was no hypertrophic lymphoglandular structures. Suspecting an ovarian teratoma, we performed an abdominopelvic magnetic resonance imaging (MRI) scan, which revealed a $20-\mathrm{cm}$ liquid mass (Figure 1) without any relation to the nearby structures and independent from the ovary-an image consistent with a foreign body. Because of contradiction in radiological findings, we scheduled the patient for explorative laparoscopic surgery with the double intent of diagnosis and treatment.

\section{Surgical Technique}

The patient was placed in the split-leg position with the legs apart and the arms abducted. The surgeon stood between the patient's legs, the assistant stood to the patient's right side, and the scrub nurse stood to the patient's left side.

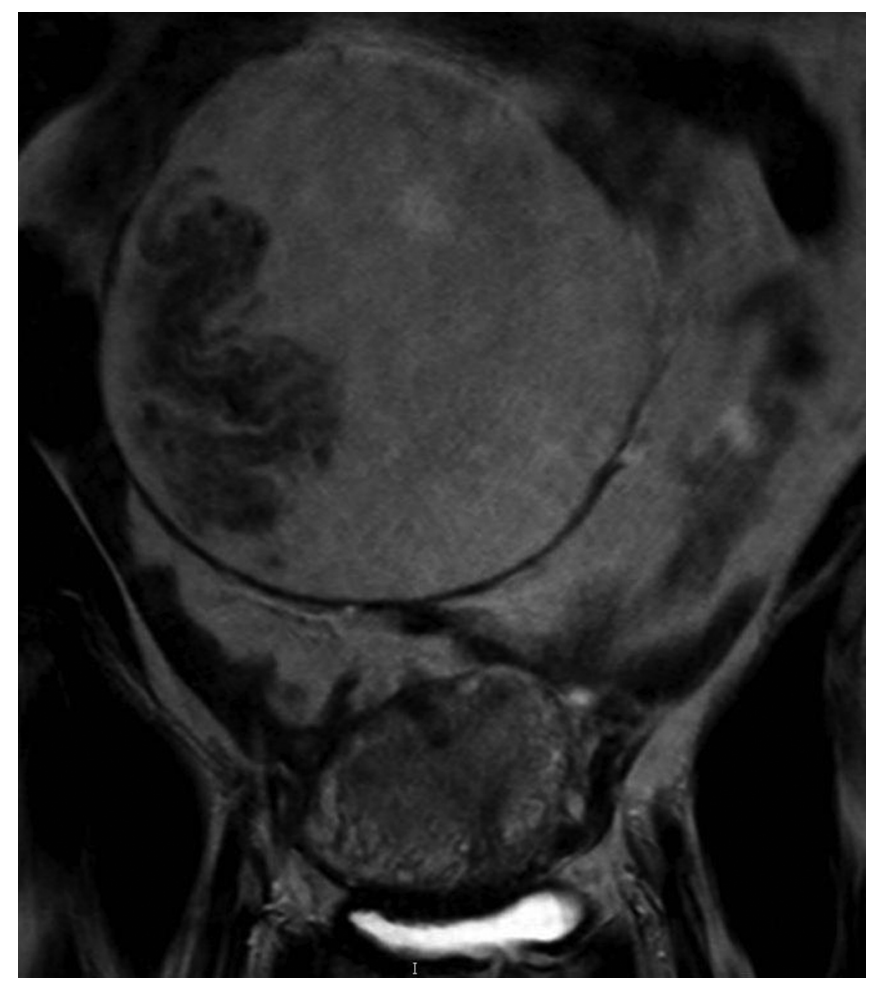

Figure 1. Abdominal mass found on magnetic resonance imaging.
A pneumoperitoneum was created by an "open laparoscopy" technique at the midline of the previous Pfannenstiel incision scar, and a 10-mm trocar for the optical system was introduced. Laparoscopic exploration of the abdominal cavity showed a floating mass in the mesogastric region, subdued to tenacious adhesions with the omentum and transverse colon and absence of both adenopathy and metastatic lesions. Two 5-mm trocars for the operative instruments were placed at the lateral edges of the Pfannenstiel incision to avoid further scarring. During the first step of the procedure, the patient was placed in the $30^{\circ}$ Trendelenburg position to obtain a wider operative field. Lysis of adhesions between the omentum and the mass was carried out with a Harmonic Scalpel (Ultracision, Ethicon Endo-Surgery Inc., Cincinnati. Ohio). Once the mobilization was completed, the patient was positioned in the $20^{\circ}$ anti-Trendelenburg position, allowing the mass to drift into the pelvic cavity. The mass was then extracted with an 8-cm laparotomy at the same level of the previous Pfannenstiel incision (Figure 2). No drain was placed. The operative time was 90 minutes and blood loss was 30 $\mathrm{mL}$. No postoperative complications occurred and the patient was discharged on the second postoperative day. At 3-month follow-up, the patient was doing well.

Pathologic examination showed that fibrotic scar tissue had developed around an endo-abdominal foreign body (laparoscopic sponge) and contained a purulent aseptic liquid with no malign cells.

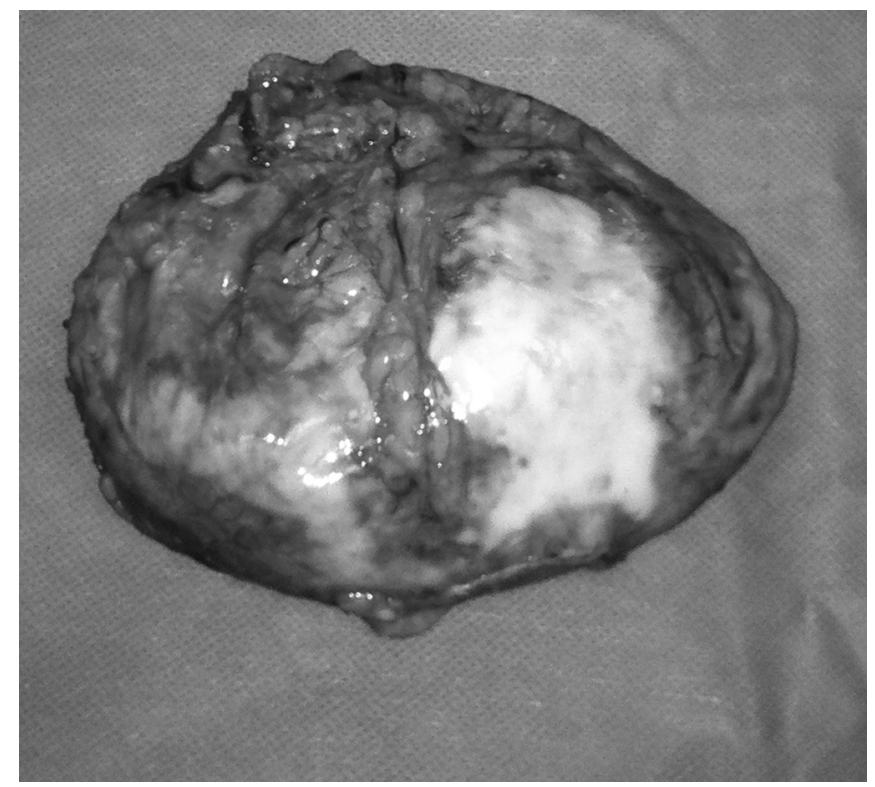

Figure 2. Gossypiboma extracted by laparotomy at the previous Pfannenstiel incision location. 


\section{DISCUSSION}

First described by Wilson et al in 1884,5 the discovery of a foreign body represents a rare occurrence in surgery, with an incidence ranging from 1 in 8801 to 1 in 18760 of inpatient operations. ${ }^{6,12}$ Although no body cavity is spared from the occurrence of a retained surgical foreign body, the abdomen and the thorax are the most frequent sites in which they are found $(74 \%$ and $11 \%$, respectively). ${ }^{6}$ Gossypiboma accounts for $69 \%$ of surgical foreign bodies, whereas in remaining cases, it is a surgical instrument such as forceps, electrodes, or retractors. ${ }^{3-8,} 14$ Virtually no surgical procedure is free from the risk of incurring this problem, as is demonstrated by several cases that occurred after laparoscopic and thoracoscopic procedures. Gawande et al identified three principal risk factors for retained sponges: emergency procedures, unplanned change in the procedure performed, and the patient's body mass index. ${ }^{6}$ The delay in the diagnosis of foreign body retention varies greatly. Singh et $\mathrm{l}^{12}$ reported a case of a sponge removal 14 days after the first operation, whereas others authors ${ }^{10}$ described a delay of up to 22 years. From pathophysiologic and clinical points of view, a gossypiboma behaves in two different ways. If the retained intraabdominal sponge has become infected or induces abscess formation, the patient is usually symptomatic within days to weeks after the initial operation. Conversely, when the sponge produces a fibrous reaction that leads to encapsulation of the foreign body, the patient shows late symptoms related to the presence of a mass. ${ }^{8,13}$ This second scenario explains the long periods with no evident clinical manifestations. ${ }^{1,9,10,14}$ As is recorded in our case, the signs and symptoms are often nonspecific and late, and exclusively linked to the increasing size of the mass and the consequent compression on nearby organs. Imaging study is essential for the diagnosis. Plain radiography can allow for identification of the radiopaque markers of the sponges, when present, but with a false-negative rate of $10 \%$ to $25 \% .{ }^{3}$ Computed tomography scan is considered to be the gold standard form of radiographic examination. It typically permits a diagnosis by detecting the aspect of the pseudotumor, the wall thickness, and a whirl-like spongiform pattern in a hypodense mass.6,13 Finally, magnetic resonance imaging has proven to be very useful in the study of a possible plane of cleavage between the mass and the surrounding structures. ${ }^{3,13}$

Once a gossypiboma is diagnosed, it should be removed, even if the patient is asymptomatic. ${ }^{4}$ Surgery via a laparotomy extraction at the site of a previous surgical incision is the preferred method of treatment for removing gossypiboma. ${ }^{1,4,14}$ However, several reports demonstrate the possibility of laparoscopic removal for small sponges, indicating the more agile lysis of adhesions and the reduced risk of bleeding as the main advantages of the mini-invasive technique. ${ }^{1,2,4,5,14}$ Furthermore, laparotomy removal of gossypibomas is not always feasible at the site of the previous operation scar, especially if it is successive to a Pfannenstiel incision, because of the frequent migration of the foreign body over the years. $3,9,13$

Enteric fistula, abscesses, pseudotumor dimensions, and long delay in diagnosis are considered contraindications to laparoscopy. 3,7,11,13 Childers et al indicated as their main criterion for the laparoscopic approach the intervention be done within a week at most from the first operation, to extract the sponge before it is encapsulated in the inflammatory reaction. ${ }^{11}$ In our patient, the laparoscopic lysis of adhesions and the complete mobilization of the mass allowed us to proceed with a little laparotomy at the site of the previous Pfannenstiel incision, avoiding both an otherwise necessary midline laparotomy incision and a new scar, and thus resulting in a better functional and cosmetic result. We believe that the time between the first operation and the removal of the sponge is not an excluding factor of the laparoscopic approach. It is evident that an operation executed within the first week offers undeniable advantages because of the absence of tenacious visceral adhesions and formation of pseudotumor that make the foreign body removal procedure more technically demanding. Yet, the use of laparoscopic ultrasonography devices like the Harmonic scalpel has made complex dissections possible with a reduced risk of bleeding and thermic damage of adjacent structures. ${ }^{15}$

In conclusion, independent of the size of a foreign body and its retention time in the body from a previous surgery, laparoscopy can be an excellent method and, when possible, should be the method of choice for gossypiboma retrieval.

\section{References:}

1. Olivier F, Devriendt D. Laparoscopic removal of a chronically retained gauze. Acta Chir Belg. 2003;103(1):108-109.

2. Târcoveanu E, Dimofte G, Georgescu S, et al. Laparoscopic retrieval of gossypibomas-short series and review of literature. Acta Chir Belg. 2011;111(6):366-369. 
3. Hariharan D, Lobo DN. Retained surgical sponges, needles and instruments. Ann R Coll Surg Engl. 2013;95(2):87-92.

4. Yuen PM, Rogers MS, Chang AM. Laparoscopic removal of retained surgical gauze after vaginal hysterectomy. Eur J Obstet Gynecol Reprod Biol. 1994;57(3):209-210.

5. Chin EH, Hazzan D, Herron DM, Salky B. Laparoscopic retrieval of intraabdominal foreign bodies. Surg Endosc. 2007;21(8):1457.

6. Childers JM, Caplinger P. Laparoscopic retrieval of a retained surgical sponge: a case report. Surg Laparosc Endosc. 1993;3(2):135138.

7. Singh R, Mathur RK, Patidar S, Tapkire R. Gossypiboma: its laparoscopic diagnosis and removal. Surg Laparosc Endosc Percutan Tech. 2004;14(5):304-305.

8. Mousavi-Bahar SH, Amirzargar MA. Laparoscopic removal of retained surgical gauze. Urol J. 2010;7(3):203-205.

9. Taçyildiz I, Aldemir M. The mistakes of surgeons: "gossypiboma." Acta Chir Belg. 2004;104(1):71-75.
10. Godara R, Marwah S, Karwasra RK, Goel R, Sen J, Singh R. Spontaneous transmural migration of surgical sponges. Asian J Surg. 2006;29(1):44-45.

11. Yuh-Feng T, Chin-Chu W, Cheng-Tau S, Min-Tsung T. FDG PET CT features of an intraabdominal gossypiboma. Clin Nucl Med. 2005;30(8):561-563.

12. Gawande AA, Fisher ES, Gruber J, Rosenthal MB. The cost of health care-highlights from a discussion about economics and reform. N Engl J Med. 2009;361(15):1421-1423.

13. Rodrigues D, Perez NE, Hammer PM, Webber JD. Laparoscopic removal of a retained intraabdominal ribbon malleable retractor after 14 years. J Laparoendosc Adv Surg Tech A. 2006; $16: 369-371$.

14. Sista F, Schietroma M, Ruscitti C, et al. New ultrasonic dissector versus conventional hemostasis in thyroid surgery: a randomized prospective study. J Laparoendosc Adv Surg Tech A. 2012;22(3):220-224. 